\title{
3D HIGH-EFFICIENCY AND HIGH-PRECISION MODEL-DRIVEN MODELLING FOR POWER TRANSMISSION TOWER
}

\author{
Zhengrong $\mathrm{Wu}^{1}$, Hao Wang ${ }^{2}$, Wenhui $\mathrm{Yu}^{1}$, Jianfei Xi ${ }^{2}$, Weigang Lei ${ }^{2}$, Tao Tang,** \\ ${ }^{1}$ China Southern Power Grid Company Limited, 510700 Guangzhou, China - (wuzr, yuwh)@csg.cn \\ ${ }^{2}$ China Southern Power Grid Digital Grid Research Institute co., Ltd, Guangzhou, China - (wanghao1, xijf, leiwg)@csg.cn \\ ${ }^{3}$ Wuhan Dynspai Technology Company Limited, Wuhan, China - dowtowne@dynspai.com
}

Commission II, WG II/3

KEY WORDS: Smart Grid; Transmission Tower; Point Clouds; 3D Reconstruction; Model-driven

\begin{abstract}
:
Constructing the transmission tower from LiDAR point clouds is a fundamental step for smart grid. However, currently the transmission tower construction method relies heavily on manual editing, which is far from the practical industrial application. This paper proposes a model-driven based method to realize 3D construction of transmission tower fast and accurately. This method first generates different types of 3D tower models. Then, it calculates the direction characteristic of point clouds distribution using the obtained transmission towers point clouds. While finding the principal direction of transmission towers, the local coordinates of the transmission towers are settled. And then the key points are captured in a semi-automatically way. According to these key points, the transmission tower model that best matches the point clouds is selected using the model matching algorithm. Comparing with the existing traditional manual editing methods, the method proposed in this paper can ensure the integrity and accuracy of the reconstructed tower model using the model-driven based strategy. The proposed method makes a trade-off between manual editing and efficiency, which guarantees the quality of tower modelling. And the feasibility and practicability of the proposed method are verified by the experiments on real-world point clouds data.
\end{abstract}

\section{INTRODUCTION}

Taking the network operation and management for power energy development into consideration, it is currently necessary to establish a smart grid system, which is characterized by informatization, automation, and interaction (Chen et al., 2009, Liu et al., 2012). The electricity transmission tower is a key component in the transmission network system, and its 3D model is a vital part of the digital and intelligent power grid. Because the transmission lines are generally long while the terrain is always complicated, especially for some power lines in complex terrain areas such as mountains and swamps, it is difficult to obtain the transmission grid data by traditional measurement methods. Light Detection And Ranging (LiDAR) based on the UAV platform has become an important technique for the acquisition of spatial-temporal data of transmission lines due to its flexible control methods and low operating costs (Ye et al., 2015, Tan et al., 2012).

\section{RELATED WORKS}

Among the 3D modelling of transmission lines, ground buildings, and terrain around power corridors, the 3D modelling of towers is not yet mature due to the diverse types and complex structures of transmission towers. Currently the transmission tower reconstruction relies heavily on manual editing, which is time-consuming and inefficient, restricting the efficiency and quality of the $3 \mathrm{D}$ modelling of the transmission line corridor. At present, the research on 3D modelling of transmission grids mainly focuses on the extraction and modelling of transmission lines. However, the research on the 3D modelling of towers is still in its infancy (Zhang et al., 2014, Yin et al., 2015, Ma et al., 2017, Zheng et al., 2017). Jwa et al., (2009) proposed a piecewise vertical curve model growth algorithm to locate the position of the power line. They clustered the power line point cloud by iteratively fitting the vertical curve and voxel growing. Guo et al., (2016) proposed an improved algorithm for reconstruction power line from complex scenarios, considering the contextual information of related pylon objects. Li et al., (2018) considered the differences in power lines and the structure of the tower, and modelled the multi-line transmission lines of the same tower in the regional power grid. Peng et al., (2019) analysed the supervised classification methods for power line inspection form airborne laser scanning system (ALS) point clouds. However, there are relatively few studies on geometric modelling of electricity power towers, and most common methods mainly count on manual modelling. For example, Yin et al., (2015) used SketchUp, a commercial software, to conduct $3 \mathrm{D}$ modelling of power transmission towers, auxiliary insulators and the armour clamp, but found that the modelling efficiency of the tower with complex structure is low. In order to improve the modelling efficiency of power transmission towers, Zheng et al., (2017) proposed a component modelling method for the 3D model of power transmission towers. The method first decomposed the input 3D models of different types of transmission towers, establishing a full-element component model base of transmission towers, and then they calculated the modelling parameters such as the spatial position and size of the components from the point clouds of the transmission grid. And by manually inputting these parameters, the component model was established, the final transmission tower model was combined according to the fixed connection relationship between different components. Although this method can meet the needs of the more detailed modelling of different types of 
transmission towers, the process of splitting the tower pole, modelling independently, and merging the sub-model into a whole model increases the workload of manual editing. At the same time, different components may be mistakenly merged, affecting the quality of the tower modelling. To tackle the problem of 3D modelling of transmission towers, this paper proposes a model-driven reconstruction method of transmission towers from laser point clouds with high efficiency and quality.

\section{METHODOLOGY}

All types of transmission line towers have explicit design specifications and manufacturing standards. Based on this, the method proposed in this paper firstly build a standard model base of towers, and then implement a model-driven approach to realize semi-automatically modelling of transmission line towers to improve the efficiency and reusability. The main method flow is shown in Figure 1. The method includes threefold: model base building, tower and transmission line marking and the model constructing based on key points capture.

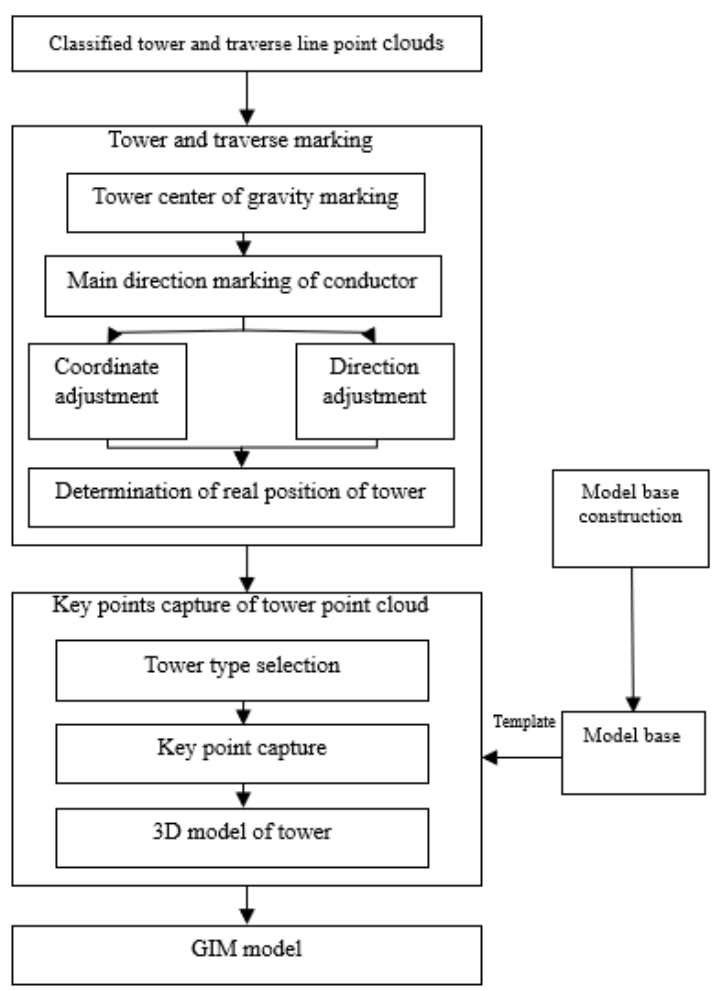

Figure 1. Methodology flowchart

\subsection{Model Base Build}

The construction of the tower model base is one of the keys to realize fast 3D modelling of transmission lines. The creation of the model base provides a template for the $3 \mathrm{D}$ modelling of the tower, improving the modelling efficiency, and decreasing the manual operations. According to the designed principles and construction standards of line towers, different types of towers have specific parameter standards. This paper builds some common types of transmission towers model and creates a group of tower model base. There are mainly 6 kinds of tower types including Drum type, Goat type, "JG" type, Cup type, Cat type and Steel pipe towers, as shown in Figure 2.

Unlike Zheng et al., (2017), who decomposed the tower model into the tower head and the tower foot, respectively created the component model and organized the model base, this paper passes through the towers in units of nodes and divides the transmission line into sections based on its slope. A top-bottom building block construction way is adopted to manually create the fine model of the predesigned transmission tower in the AutoDesk CAD software.

The tower model base is built by level-wise building blocks. Thus, the height of transmission tower body is changed while keeping the type of the tower head unchanged by adjusting parameters. Based on the above description, the modelling method used in this paper could easily construct towers with different height while keeping the same tower head unchanged.

\subsection{Tower and Transmission Line Marking}

This paper proposes a model-driven method for constructing 3D model of transmission tower. Therefore, to construct highprecision model of the tower in the transmission line corridor, the tower and the line need to be marked to obtain the coordinates and direction of the tower model, and to determine the spatial position of the tower model further. It's obvious that the tower and transmission line marking accuracy determinates the final model's precision. So, the process of marking the tower is particularly important. This paper proposes a dimension-feature based approach for marking the tower and the corresponding power line.

Firstly, according to the classified point clouds of tower and the traverse line, the centre of gravity $\left(X_{\mathrm{g}}, Y_{g}\right)$ of each tower is calculated according to equations (1) and (2), and the centre of gravity is treated as the origin of each tower independent coordinate system;

$$
\begin{gathered}
X_{\mathrm{g}}=\frac{\sum_{i=1}^{M} x_{i}}{M} \\
Y_{\mathrm{g}}=\frac{\sum_{i=1}^{M} \mathrm{y}_{i}}{M}
\end{gathered}
$$

where $\quad x_{\mathrm{g}}, \mathrm{y}_{g}$ are the coordinate of the point this tower.

$$
\mathrm{M} \text { is the total number of point clouds belonging to }
$$

Then, according to the lowest attitude $Z_{\min }$ of the point clouds, the bounding box is easily obtained, which contains the whole point clouds belonging to this tower. And the local independent coordinate can be converted based on the centre of the gravity and the bounding box, as shown in Figure 3 .

The point cloud set belonging to the identity transmission line are grouped by the point-wise dimension feature and region growing. To be specifically, based on the dimension feature distribution, space and semantic information could be used to cluster the point clouds together. Mathematically, by setting a certain radius, the neighbourhood point cloud could be formulated into a covariance matrix and the feature in each $\mathrm{x}, \mathrm{y}$, $\mathrm{z}$ dimension could be inferred. The covariance feature values $\lambda_{1}, \lambda_{2}, \lambda_{3}\left(\lambda_{1}>\lambda_{2}>\lambda_{3}\right)$ are formulated to represent the dimension feature by equation 3 :

$$
\left(a_{1 D}, a_{2 D}, a_{3 D}\right)=\left(\frac{\sqrt{\lambda_{1}}-\sqrt{\lambda_{2}}}{\sqrt{\lambda_{1}}}, \frac{\sqrt{\lambda_{2}}-\sqrt{\lambda_{3}}}{\sqrt{\lambda_{1}}}, \frac{\sqrt{\lambda_{3}}}{\sqrt{\lambda_{1}}}\right)
$$

Where $a_{1 D}+a_{2 D}+a_{3 D}=1$ 


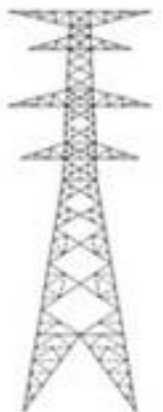

(a) Drum type tower

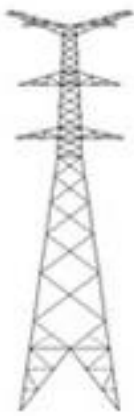

(b) Goat type tower

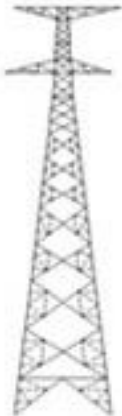

(c) "JG" type tower

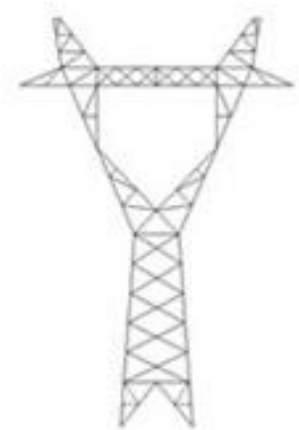

(d) Cup type tower

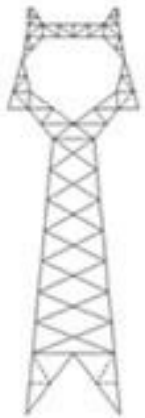

(e) Cat-head tower

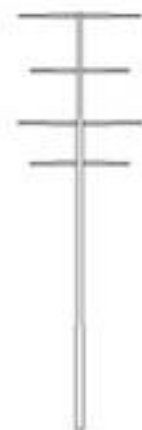

(f) Steel pipe tower

Figure 2. Model base construction

The power line point appears as a line-like object in geometry space and has a linear spatial distribution characteristic. Mathematically, one of the eigenvalues of the k neighbourhood point cloud in a local tangent plane will be larger than the other two eigenvalues, e.g. $\lambda_{1} \gg \lambda_{2} \approx \lambda_{3}$, According to this distribution characteristic of the point clouds, the point-wise dimension feature could be calculated. And the region grown algorithms is used to cluster the point with the same feature distribution characteristic through randomly selecting a line-like power line point as seed (Chen et al., 2015). Finally, the point clouds are separated into different sets that each set contains only one power line and the point cloud in each set has similar principal direction. Based on the separated point cloud set, the principal direction of the current power line is used to define the traverse line path and the tower local coordinate's principal direction. As shown in Figure 4 (a), the $\mathrm{X}$ axis, rendered as green line, is set to align with the traverse line direction and the $\mathrm{Y}$ axis, rendered as red line, is orthogonal to the green line.

Finally, according to the bounding box of each tower and the corresponding principal direction of the traverse line, the coordinate of each tower are adjusted to determine the tower model's pose in real point cloud space, as shown in Figure 4(b) and Figure 4 (c).

\subsection{Tower Model Construction Based on key-point Capture}

After tower and transmission line marking, the pose of the tower model is obtained. This paper adopts an interactive way to construct the tower model by manually capturing the key point from point clouds scanning on the real tower. More details are as following steps:

1) A curve surface is represented by one point in tower and its $n$ neighbourhood point clouds are formulated by equation 4 . And the surface parameters are calculated by least square method. Thus, the local curvature $\mathrm{K}$ is shown in equation 5 :

$$
\begin{gathered}
Z(x, y)=A x^{2}+B y^{2}+C x y+E y+F \\
K=\frac{A\left(1+E^{2}\right)+B\left(1+D^{2}\right)-C D E}{\left(1+D^{2}+E^{2}\right)^{\frac{2}{3}}}
\end{gathered}
$$

where $\quad(x, y)$ are the coordinate of point cloud

$\mathrm{A}, \mathrm{B}, \mathrm{C}, \mathrm{D}, \mathrm{E}, \mathrm{F}$ are the parameters of local curve surface.
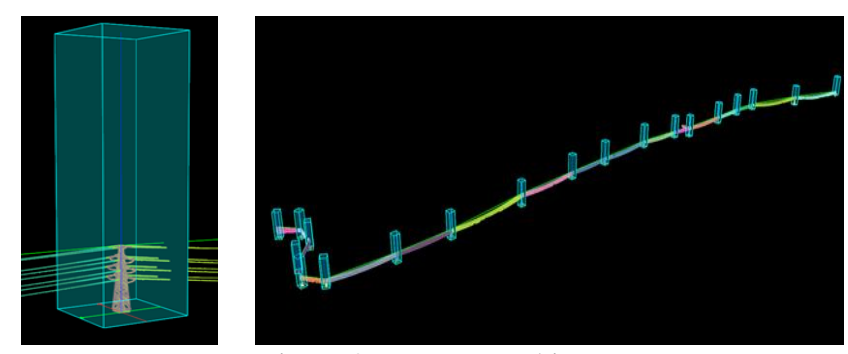

Figure 3. Towers Marking

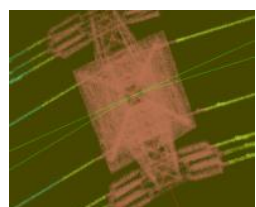

(a) path defined by principal directions of lines

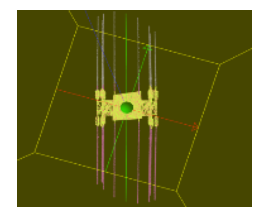

(b) before coordinate system adjustment

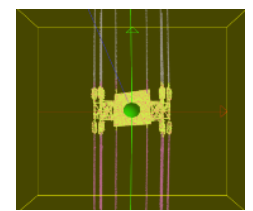

(c) after coordinate system adjustment
Figure 4. Positions and path tracks of towers

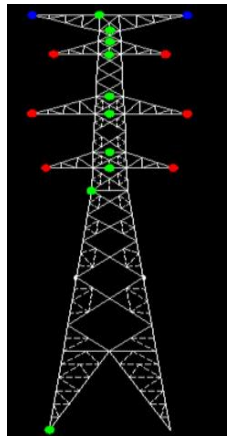

(a) Caputuring of key points

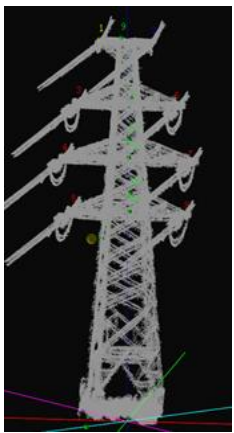

(b) Visualization of key points

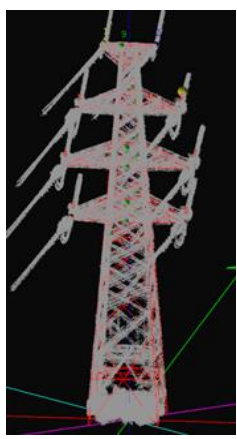

(c) Construction of tower models
Figure 5. Construction of tower models based on capturing of key points

2) Capturing the largest curvature points as key-points, which are shown in red, green, blue bold points in Figure 5 (a). The keypoint of a tower are divided as three types including the earth wire points shown as blue points, traverse points shown as red points and the tower body points shown as green points. All key points should be captured correctly in an interactive way, especially the earth wire points and the traverse points. Because this kind of points describes the whole outline of the tower and makes a foundation to the further model matching. 
3) According to the captured key points' location and the tower model base, the model stored in the data base whose outline is similar to the current tower key points' outline is selected. The type of model that minimize the sum of distance of each key points and the corresponding points in model base is returned. The model matching is simply done by stretching this model in vertical direction to align the model with the key points. The tower model is constructed through key points capturing and model matching.

Besides, the models are constructed by piece of building blocks, the height of tower model could be adjusted easily by selecting the key points in cross arm and measuring the length of different tower along the $\mathrm{Z}$ axis, as shown in Figure 6 . Modifying the standard reference model, the variable height transmission tower is built with the same tower head.

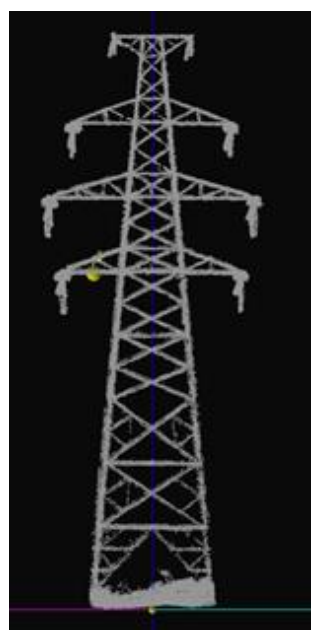

(a) Capture the lowest crossarm point

Figure 6. Generation of reference tower

\section{EXPERIMENTS}

\subsection{Dataset}

The model construction performance is tested on Windows 10 64-bit laptop (Intel7 8 core, 8G memory), using OpenCV, PCL open source library and AutoCAD commercial software to implement and build the transmission tower model base. This paper takes an actual high-voltage transmission line corridor as an example, which contains 19 towers. There are only two types of tower in this area, the cat-head type and the "JG" type, as shown in Figure 7 (a, c). Besides, they are the most common types of towers. Therefore, this paper mainly models and analyses these two types of towers. The Laser Scanning point clouds are acquired through an onboard LiDAR laser scanning system, and the average point density of the point cloud is 10 points $/ \mathrm{m}^{2}$. The ground point is filtered using the method proposed by Yang et al., (2016). Following the method mentioned in section 3.2, the point clouds are classified as tower points and transmission line points in which each point has a point-wise semantic label, for further tower modelling.

The tower-ground connection point and the centre of gravity of the classified tower point cloud are calculated and used to locate the local coordinate origin. And the dimension feature is used to determine the orientation of the tower model and traverse line's principal direction. Via key points capturing from one tower to another tower, the whole transmission line corridor's model is constructed in a short time.
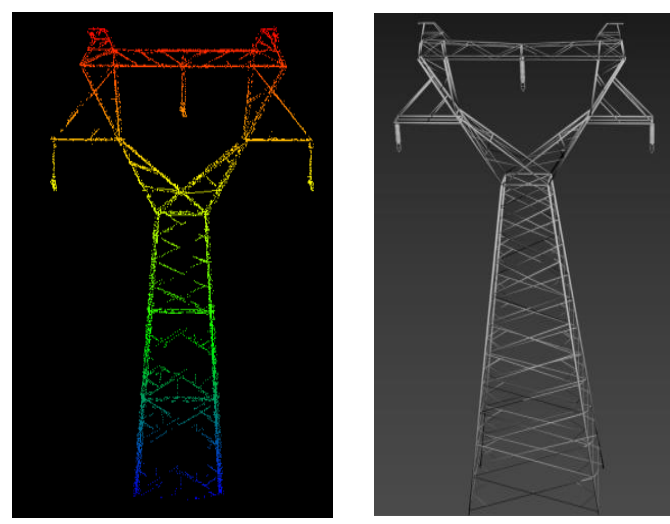

(a) Point cloud of tower 1

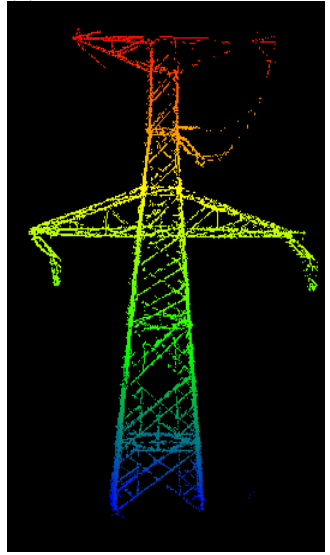

(c) Point cloud of tower 2
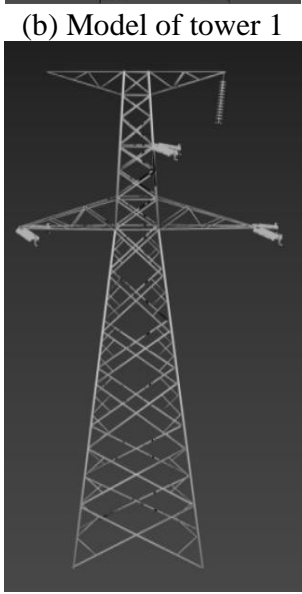

(d) Model of tower 2

Figure 7. Comparison of original point clouds of a given power transmission tower and its 3D modelling

During the model-driven modelling, any certain types of tower could be used to form the tower model template base. The absolute geometry information of tower such as cross arm, tower head and tower root's location are obtained from the key points in real point clouds lying on these positions. Meanwhile, the whole approach has been integrated into a 3D modelling engine platform. The model could be rendered real-time which is convenience for capturing the precision position and accurate model construction. Figure 7 shows two tower models and their corresponding origin point clouds. It can be seen that the detailed structures are maintained intuitively.

\subsection{Quantitative Results}

The time-consuming and accuracy comparison results are listed in Table 1. The method proposed in this paper reduces the time cost to tenth of the traditional CAD tower modelling method, significantly improving the efficiency.

In this paper, the number of towers in this test area is 19 , including 14 double-circuit towers and 5 steel pipe pole towers. The input data to CAD and the approach proposed in this paper are accomplished without any pre-process. The key points lying on the cross arm and tower body are captured to ensure the similar outline of the tower. Single Drum-type tower requires to capture 56 key points and connect almost 100 bars. The whole test transmission line requires to capture around 800 to 1000 key points and connect 1500 bars, taking about 600 minutes. The approach in this paper only requires to capture 297 key points theoretically per tower. However, given the fact that the reference tower with the same tower head doesn't need to input again, the total captured key points are cut to 144 with no 
connecting bars. It has to be noted that the key points are automatically highlighted in the operation window, which also improves the efficiency.

Compared with the key points' location precision selected by an expert operator, the key points automatically extracted in this paper are accurate enough under the error tolerance. As listed in Table 1, the traverse hanging point, the end point of insulator and the end point of tower header are chosen to verify the keypoint selected precision. The key points' position captured by expert operator are treated as ground truth, and the Euclidean distance error is calculated between the ground truth and the key points' position extracted automatically in this paper. The approach proposed in this paper makes a trade-off between the efficiency and the accuracy during the transmission tower construction.

The construction of each transmission tower is a repeat process as the description mentioned in Section 3. And more construction results are shown in Figure 8. Figure 8 shows the $3 \mathrm{D}$ visualization of experimental tower model in three different areas. As results show, in a large field of transmission power corridor, in terms of construction efficiency, the method proposed in this paper could satisfy the demand of transmission tower modelling with high precision.

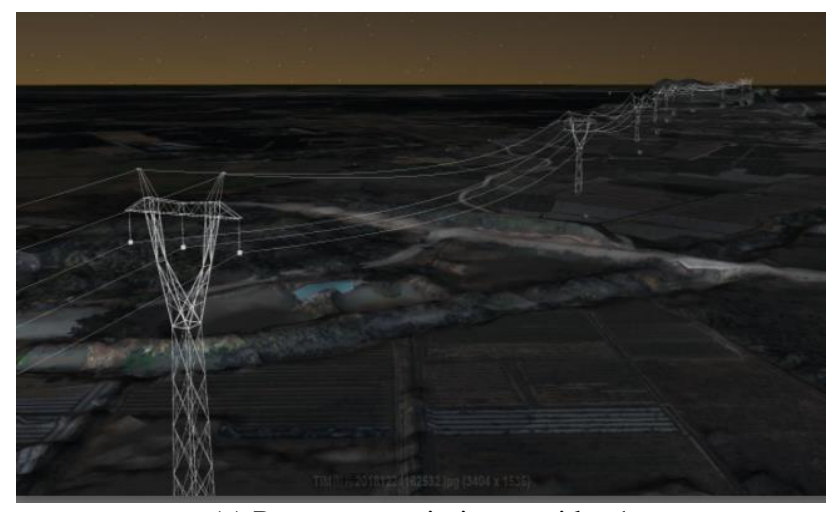

(a) Power transmission corridor 1

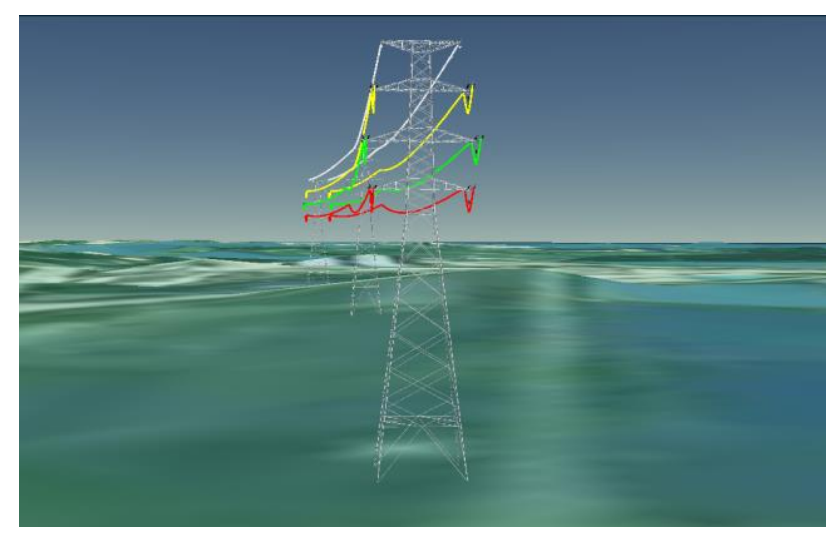

(b) Power transmission corridor 2

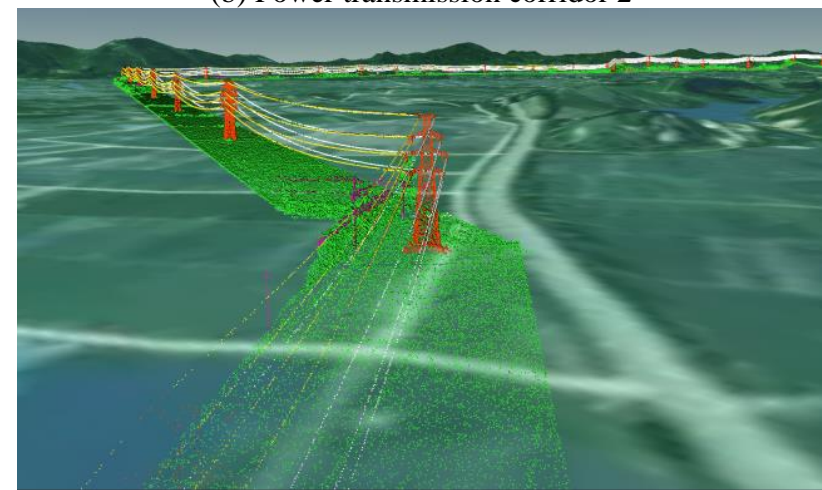

(c) Power transmission corridor 3

Figure 8 . The 3D visualization of power transmission lines. It can be seen that the model constructed in this paper is satisfied with the real data.

\section{CONCLUSIONS}

Currently, the construction of transmission tower and traverse line in a large area is labor intensive and time consuming. This paper proposes a semi-automatic model-based transmission tower modelling method from LiDAR point clouds to solve the problem of low efficiency. The input data are the transmission line point cloud and the several pre-build transmission tower models. The tower's principal direction and its local coordinate

\begin{tabular}{|c|c|c|c|c|c|c|c|}
\hline \multirow{2}{*}{$\begin{array}{l}\text { Modelling } \\
\text { method }\end{array}$} & \multicolumn{2}{|c|}{$\begin{array}{l}\text { Modelling time of single } \\
\text { tower / min }\end{array}$} & \multicolumn{2}{|c|}{$\begin{array}{l}19 \text { tower modelling time / } \\
\text { min }\end{array}$} & \multicolumn{3}{|c|}{$\begin{array}{l}\text { Average error of selecting key points of } 19 \\
\text { towers (compared with CAD manual modelling) } \\
\qquad / \mathrm{m}\end{array}$} \\
\hline & $\begin{array}{c}\text { Capture } \\
\text { key points }\end{array}$ & Connect bar & $\begin{array}{c}\text { Capture } \\
\text { key points }\end{array}$ & Connect bar & $\begin{array}{c}\text { Traverse } \\
\text { hanging point }\end{array}$ & $\begin{array}{l}\text { End point of } \\
\text { insulator }\end{array}$ & $\begin{array}{l}\text { End point of } \\
\text { tower head }\end{array}$ \\
\hline $\begin{array}{l}\text { CAD } \\
\text { modelling } \\
\text { Method } \\
\text { proposed in } \\
\text { this paper }\end{array}$ & 10 & 20 & 200 & 400 & 0.381 & 0.335 & 0.258 \\
\hline
\end{tabular}

Table 1. Comparison of tower modelling time. The time consuming of modelling single tower

are confirmed by calculating the dimension feature of point cloud distribution. Then the model template is matched to the interactively selected key points. This model-driven based method could improve the completeness and precision of the tower modelling. Compared with human editing and component-based modelling methods, it requires less human editing but guarantees the efficiency and the model quality. However, a relatively complete transmission tower base is in assumption, and the generalization of the method counts on the types of tower stored in the base. In the future, a more automatic and accurate method will be studied.

\section{ACKNOWLEDGEMENTS}

This research is supported by Science and technology project of China Southern Power Grid Corporation (ZBKJXM20170229). 


\section{REFERENCES}

Chen, C., Mai, X., Song, S., Peng, X., X. W., Wang, K., 2015. Automatic power lines extraction method from airborne lidar point cloud. Geomatics and Information Science of Wuhan University, 40(12):1600-1605. doi.org/10.13203/j. whugis2013 0573 .

Chen, S., Song, S., Li, L., Shen, J., 2009. Survey on smart grid technology. Power System Technology, 33(8), 1-7.

Guo, B., Li, Q., Huang, X., Wang, C. 2016. An improved method for power-line reconstruction from point cloud data. Remote Sensing, 8(1), 36. doi.org/10.3390/rs8010036.

Jwa, Y., Sohn, G., 2009. Automatic 3D powerline reconstruction using airborne LiDAR data. International Archives of Photogrammetry Remote Sensing and Spatial Information Sciences 38 (Part 3/W8), 105-110.

Li, X., Li, F., Liu, P., Cai, W., Cai, Z. 2018. Modelling approach for short-transmission lines on the same tower with different wire parameters and tower structure. 2018 International Conference on Power System Technology. doi.org/10.1109/powercon.2018.8601542.

Liu, W., Yang, H. X., Zhu, B., 2012. Review of research on smart grid technical standard system. Power System Protection and Control, 40(10), 120-126. doi.org/10.3969/j.issn.16743415.2012.10.022.

Ma, Q, 2017: Research on UAV power inspection and 3D model reconstruction technology. Shandong University China. Peng, S., Xi, X., Wang, C., Dong, P., Wang, P., Nie, S. 2019. Systematic comparison of power corridor classification methods from als point clouds. Remote Sensing, 11(17), 1961. doi.org/ $10.3390 / \mathrm{rs} 11171961$.

Tan, J., Liu, L., Zu, W., 2012. Power line distance measurement method in helicopter power line inspection. Science of Surveying and Mapping (3), 63-65. doi.org/cnki:sun:chkd.0. 2012-03-020.

Yang B., Huang R., Dong Z., et al., 2016. Two-step adaptive extraction method for ground points and breaklines from lidar point clouds. ISPRS Journal of Photogrammetry and Remote Sensing, 119, 373-389. doi.org/10.1016/j.isprsjprs.2016.07.002.

Ye, Q., Wu, D., Li, W., 2015. Powerline danger point detection using uav lidar point cloud. Bulletin of Surveying and Mapping, 000(11), 57-59. doi.org/ 10.13474/j.cnki.11-2246. 2015.0346.

Yin, H., Sun, M., Gan, Z., Zhang, X., Huang, S., 2015. 3D modelization of transmission tower based on sketchup. Bulletin of Surveying and Mapping(4), 34-37. doi.org/10.13474/j.cnki. 11-2246.2015.0106.

Zhang, W., Peng, X., Zhong, Q., Chen, R., Liu, Z., Zuo, Z., Luo, Z., 2014. Status quo and expectation of safety inspection technology for electric power lines based on remote sensing. Guangdong Electric Power, 000(002), 1-6. doi.org/10.3969/j. issn.1007-290X.2014.02.001.

Zheng, X., Chen, C., Wang, K., Song, S., Qian, J., Yang, B., 2017. 3D high-efficiency and high-precision modeling for power transmission tower based on all-factor component model.
Guangdong Electric Power, 030(6), 94-97,131. doi.org/10.134 74/j.cnki.11-2246.2015.0106. 